\title{
A Rare Cause of Melaena-Duodenal Gangliocytic Paraganglioma
}

\author{
Carmencita Esquivel, Suresh Navadgi, Greg Otto, Rebecca Thomas* \\ Department of Surgery, Lyell McEwin Hospital, Elizabeth Vale, Australia \\ Email: "beccathom@yahoo.com
}

Received September 29, 2011; revised December 22, 2011; accepted January 5, 2012

\begin{abstract}
Gangliocytic paraganglioma (GP), a rare tumour that arises most frequently from the periampullary area, is considered to be a benign neoplasm with a potential for lymphatic spread. Distant metastases are rare [1]. We report a case of a 51 year old female who presented with abdominal pain, anaemia and melaena. Endoscopy, push enteroscopy and biopsy revealed a periampullary neuroendocrine tumour. Immunohistochemistry of the pancreatic duodenectomy specimen demonstrated a duodenal gangliocytic paraganglioma with no lymph node metastases. We review the literature on this rare tumour and the current treatment protocol.
\end{abstract}

Keywords: Melaena; Rare Tumour; Neuroendocrine Tumour; Diagnostic Histological Triad; Gangliocytic Paraganglioma

\section{Introduction}

Gangliocytic paraganglioma (GP) is an uncommon tumour of the gastrointestinal tract with majority of cases arising in the duodenum [2]. Other reported sites include the jejunum, pylorus, oesophagus, pancreas and appendix [3]. GP is generally benign with a low potential for lymph node and distant metastases.

We report a case of duodenal GP which has been treated with pancreaticoduodenectomy.

\section{Case Presentation}

A 51 year old post menopausal Asian female presented with vague abdominal pain and recurrent melaena. After initial normal panendoscopies, a capsule endoscopy revealed a submucosal mass in the distal duodenum (Figure 1). A push enteroscopy then revealed a $2 \mathrm{~cm} \times 3.5$ $\mathrm{cm}$ periampullary lesion with a central ulceration (Figure 2). Immunocytochemistry of the enteroscopy fragments were synaptophysin positive but negative for chromogranin and S 100, supporting an impression of a neuroendocrine tumour.

Pre-operative ERCP showed a localised periampullary mass. Staging computerised tomograms of the chest, abdomen and pelvis were unremarkable. Octreotide scan was negative. Serum chromogranin was normal.

The patient underwent an uneventful pancreaticoduodenectomy. She is for annual follow up with CT scan and

"Corresponding author.

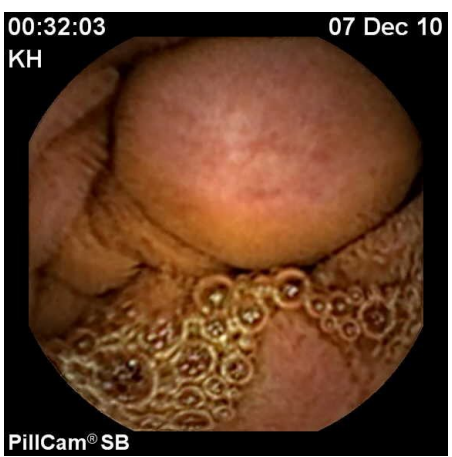

Figure 1. Capsule endoscopy-duodenal submucosal mass.

chromogranin A studies.

\section{Histopathology}

The gross specimen included a $180 \mathrm{~mm}$ segment of the duodenum and the head of the pancreas with a periampullary pedunculated firm tumour measuring $35 \times 15 \times$ $15 \mathrm{~mm}$ and five benign peripancreatic lymph nodes. Microscopically, the tumour was confined to the duodenal submucosa (Figure 3(a)) and was composed of uniform epithelial cells showing trabecular and nested growth pattern, groups of spindle cells (Figure 3(b)) and islands of ganglion cells. (Figure 3(c)). No lymphovascular invasion was identified. The tumour cells stained positive for chromogranin (Figure 4) and CD56 (Figure 5). The spindle cell component was positive for S100 (Figure 6). These findings are consistent with a gangliocytic para- 


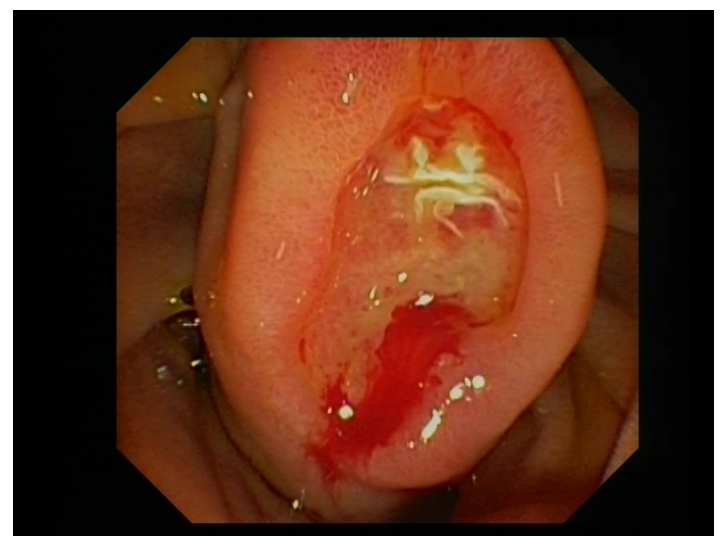

Figure 2. Push enteroscopy-2.5 $\times 3.5 \mathrm{~cm}$ periampullary lesion with central ulceration.

ganglioma.

\section{Discussion}

Gangliocytic paraganglioma (GP) is a rare benign neuroendocrine tumour, majority being duodenal [4], in a mean age group of 52 to 54 years, with no significant gender difference [5].

The most common presenting symptom is gastrointestinal bleeding [5]; the other being abdominal pain. Biliary and duodenal obstruction is rare [6].

\subsection{Histology}

Grossly, GP may present as single polypoid, sessile, pedunculated or multiple masses [2]. Tumour size ranges from 5.5 to $100 \mathrm{~mm}$ [5].

GP is usually confined to the submucosa but may infiltrate smooth muscle and ampullary ducts.

The triad of epithelioid cells, ganglion cells and spindle cells is pathognomic of GP. Several theories have been cited to explain the co-existence of these three cell types. One theory suggests that GP arises from pleuripotent stem cells at the bottom of duodenal crypts $[7,8]$. Alternatively, the tumour may originate from pancreatic epithelioid cells and the retroperitoneal elements of ganglion and spindle cells [7].

Immunohistochemical studies help to identify each cell type. Ganglion cells stain positive for synaptophysin, CD 56 and neuron specific enolase (NSE). Epithelioid cells show positive staining for chromogranin A, NSE, CD 56 and pancreatic polypeptide. Spindle cells stain with S 100 protein (8).

The predominance of each of the three cell types in GP may vary.

\subsection{Metastases}

Despite the benign nature of GP, it has a 5\% [9] to 6.9\% [5] potential for lymph node metastases.

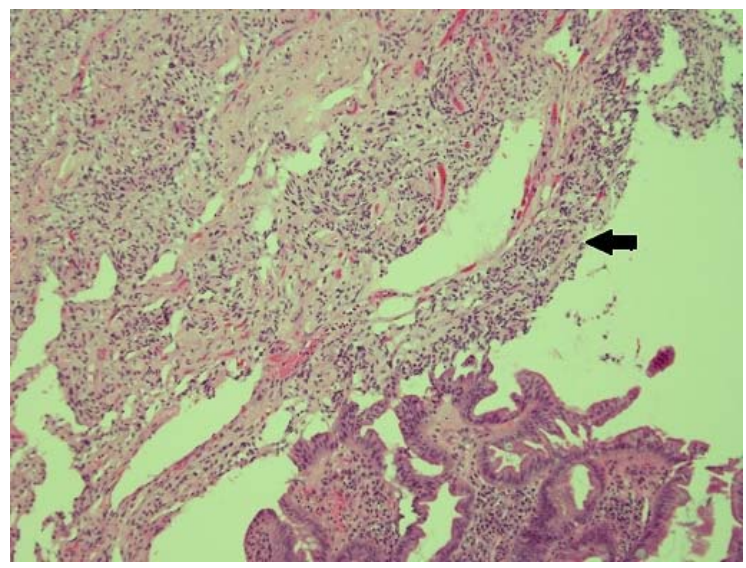

(a)

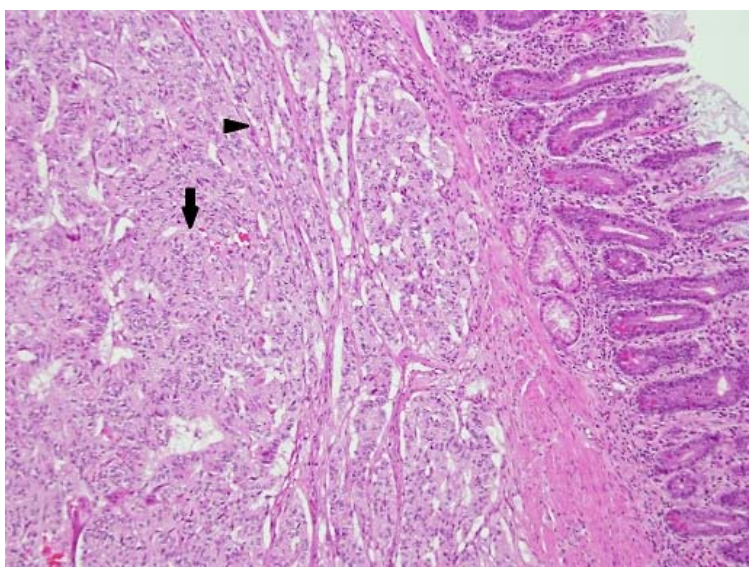

(b)

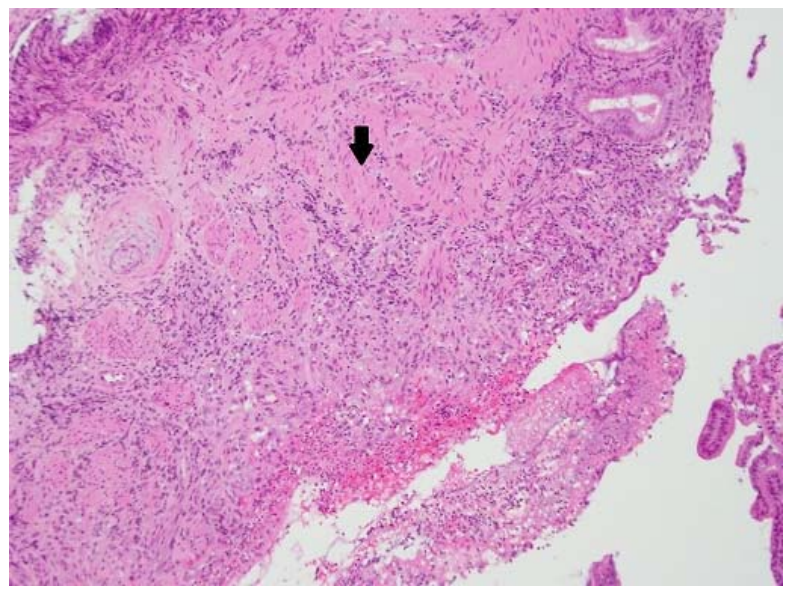

(c)

Figure 3. (a) Haematoxylin and eosin (H \& E) stainingsubmucosal infiltration of the tumor cells (arrow); (b) H \& E Epithelial cells in trabeculae and nested growth pattern (arrow) with groups of spindle cells (arrowhead); (c) H \& E-Islands of ganglions cells in submucosa (arrow).

Tumour size does not correlate with LN metastases $[10,11]$. Metastatic potential rises with increased rate of mitosis and spread into the muscularis propria [11]. It is 


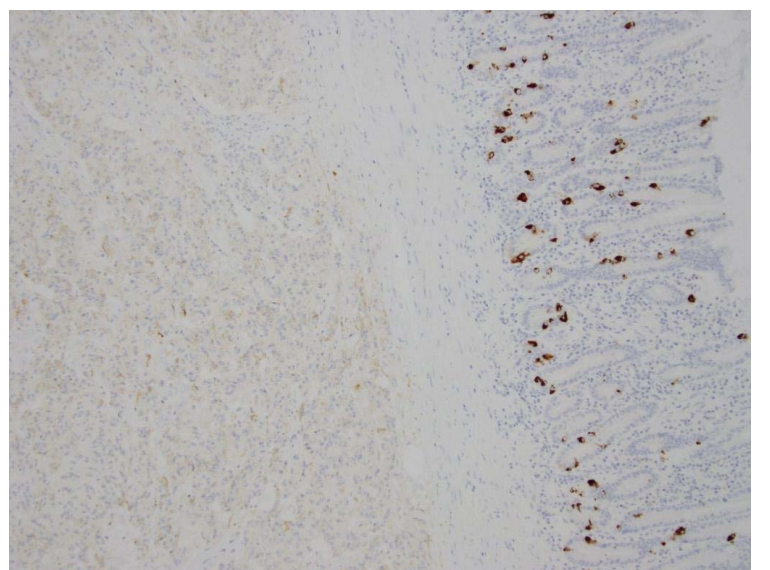

Figure 4. Epithelial cells weakly stained positive for chromogranin.

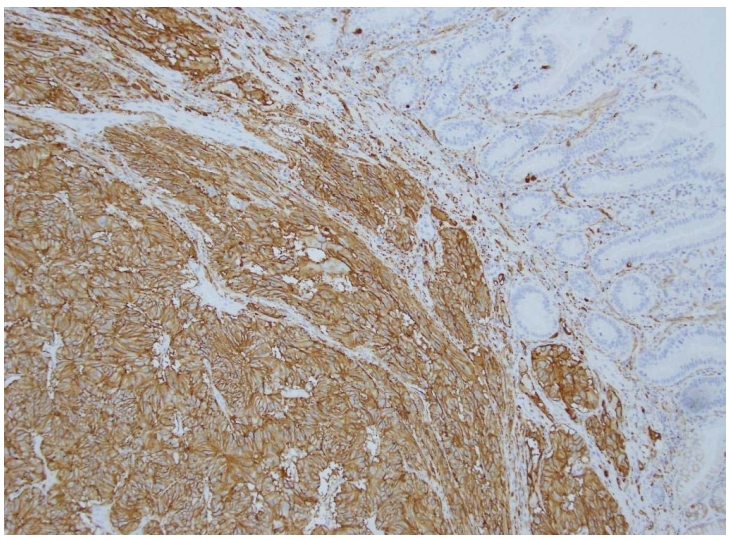

Figure 5. Positive staining to CD 56 in epithelial and ganglion cells.

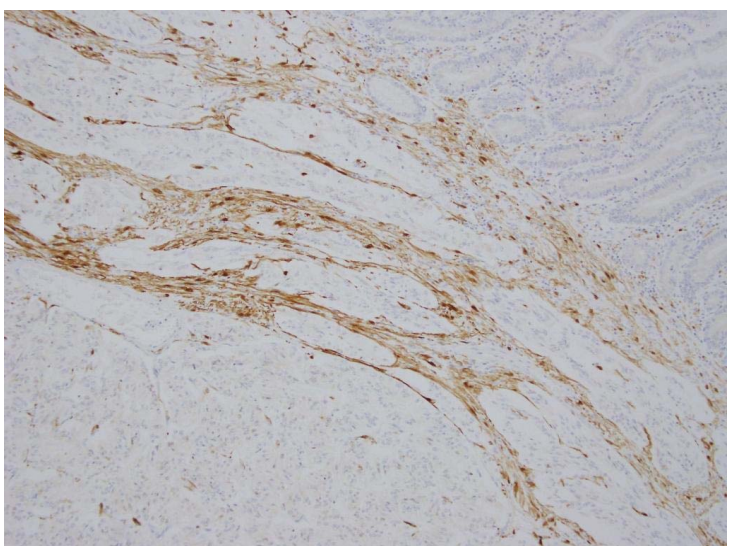

Figure 6. Spindle cells showing positive staining to S 100 protein.

of significance to note that most cases of duodenal GP with lymph node involvement are manifested by spread of the epithelioid component only [12].

Okubo et al described the demographic profile of patients with metastatic GP. Patients with lymph node me- tastases were younger than those without. Higher rates of submucosal invasion and lymph node metastases were found in female patients. Furthermore, the same authors hypothesise that tumour progression may be related to sex hormone activity. This is supported by findings of increased progesterone receptors in epithelioid components of primary and metastatic foci [5].

\subsection{Work Up}

Endoscopic biopsy may be non contributory because of the submucosal nature of GP [13]. Hence, imaging is necessary for pre-operative diagnosis. Reports document the use of endoscopic ultrasound [14], ERCP and CT to confirm the extent of duodenal wall, hepatobiliary and lymphatic involvement.

\subsection{Treatment and Follow Up}

In reported cases, patients who underwent pancreaticoduodenectomy have better outcomes on follow up [13]. Pancreaticoduodenectomy maybe curative even with lymph node metastases. Local ampullectomy or endoscopic resection alone does not allow for evaluation of lymph node status.

In contrast, Kwon et al report that mass excision may be sufficient in most cases [10]. Endoscopic resection is recommended for small, localised tumours without pancreatic or biliary extension or lymphatic spread [14]. Larger tumours or suspicion of lymph node involvement benefit from pancreaticoduodenectomy with lymph node dissection.

\subsection{Adjuvant Treatment}

The role of adjuvant treatment in locally metastatic GP remains debatable, given its low likelihood of distant metastases and the curative effect of radical resection [15]. Following pancreaticoduodenectomy, follow up imaging is recommended to check for recurrence. No strict guidelines for the frequency of imaging have been reported.

There is limited use for prognostic immunohistochemistry markers like p53 or bcl-2 expression [15]. Although serum chromogranin A monitoring has not been documented to be part of standard follow up in duodenal GP per se, it is beneficial in follow up of neuroendocrine tumours [16].

\subsection{Prognosis}

Despite its potential to metastasise to lymph nodes and distant organs, GP has a favourable prognosis. There have been no reported recurrences or deaths in cases followed up after appropriate resection [5]. 


\section{Conclusions}

Gangliocytic paragangliomas are rare benign tumours of the GI tract, particularly the duodenum, with a low potential for metastasis to lymph nodes. The submucosal nature of this tumour may render diagnosis difficult by endoscopic biopsies alone. Hence, imaging is important to define its extent and spread. Suspected lymph node metastases mandate radical resection. Prognosis is excellent in majority of cases.

We have presented a case of an Asian female with typical features of duodenal GP.

Although there has been no reported racial predilection for this tumour, it is interesting to note that numerous articles are from Japanese and Korean publications. It would be worthwhile to investigate further regarding the incidence of GP with respect to ethnicity.

\section{Acknowledgements}

Dr. Georgina England, Pathology Department, Lyell McEwin Hospital.

Mr. Tim Rogers, Pathology Department, Lyell McEwin Hospital.

Mr. Pete Collins, Gastroenterology Department, Lyell McEwin Hospital.

\section{REFERENCES}

[1] C. Henry, H. Ghalel-Mechaoui, N. Bottero, T. Pradier and H. Moindrot, "Gangliocytic Paraganglioma of the Pancreas with Bone Metastasis (French)," Annales de Chirurgie Vol. 128, No. 5, 2003, pp. 336-338. doi:10.1016/S0003-3944(03)00097-X

[2] G. Altavilla, S. Chiarelli and A. Fassina, "Duodenal Periampullary Gangliocytic Paraganglioma: Report of Two Cases with Immunochemical and Ultrastructural Study," Ultrastructural Pathology, Vol. 25, 2001, pp. 137-145. doi:10.1080/019131201750222220

[3] A. Witkiewitz, A. Galler and C. Yeo, "Gangliocytic paraganglioma: Case Report and Review of Literature," Thomas Jefferson University Department of Surgery Faculty Papers and Presentations, Paper 9, 2007.

[4] A. P. Burke and E. B. Helwig, "Gangliocytic Paraganglioma,” American Journal of Clinical Pathology Vol. 92, No. 1, 1989, pp. 1-9.

[5] Y. Okubo, M. Wakayama, T. Nemoto, K. Kitahara, H. Nakayama, K. Shibuya, T. Yokose, M. Yamada, K. Shomodaira, D. Sasai, T. Ishiwatari, M. Tsuchiya and N. Hiruta, "Literature Survey on Epidemiology and Pathology of Gangliocytic Paraganglioma,” BMC Cancer, Vol. 11, 2011, p. 187. doi:10.1186/1471-2407-11-187

[6] C. M. Mann, S. R. Bramhall, J. A. Buckels and P. Taniere, “An Unusual Case of Duodenal Obstruction-Gangliocytic
Paraganglioma," Journal of Hepatobiliary and Pancreatic Surgery, Vol. 16, No. 4, 2009, pp. 562-565. doi:10.1007/s00534-009-0092-8

[7] R. J. Reed, P. J. Caroca and J. C. Harkin, "Gangliocytic Paraganglioma,” American Journal of Surgical Pathology, Vol. 1, No. 93, 1977, pp. 207-216.

doi:10.1097/00000478-197709000-00002

[8] Y. Ohtsuki, R. Watanabe, M. Kimura, T. Okamoto, S. Murakami, Y. Mizukami, M. Takeji, Y. Okada, Y. Hayashi, G. Lee and M. Furihata, "Immunohistochemical and Electron Microscopic Studies of a Case of Duodenal Gangliocytic Paraganglioma,” Medical Molecular Morphology, Vol. 42, No. 4, 2009, pp. 245-249. doi:10.1007/s00795-009-0442-2

[9] A. Wong, A. Miller, J. Metter and C. Thomas, "Locally Advanced Duodenal Gangliocytic Paraganglioma Treated with Adjuvant Radiotherapy: Case Report and Review of the Literature," World Journal of Surgical Oncology, Vol. 3, 2005, p. 15. doi:10.1186/1477-7819-3-15

[10] J. Kwon, S. E. Lee, M. J. Kang, J.-Y. Jang and S.-W. Kim, "A Case of Gangliocytic Paraganglioma in the Ampulla of Vater,” World Journal of Surgical Oncology, Vol. 8, 2010, p. 42. doi:10.1186/1477-7819-8-42

[11] H. Witzigmann, C. Loracher, F. Geissler, T. Wagner, A. Tanaapfel, D. Uhlmann, K. Caca, J. Hauss and J. A. Hehl, "Neuroendocrine Tumors of the Duodenum," Langenbeck's Archives of Surgery, Vol. 386, No. 7, 2002, pp. 525-533. doi:10.1007/s00423-001-0260-Z

[12] V. Sundarajan, T. Robisnon-Smith and A. Lowy, "Duodenal Gangliocytic Paraganglioma with Lymph Node Metastasis,” Archives of Pathology \& Laboratory Medicine, Vol. 127, 2003, pp. e139-e141.

[13] P. Bucher, Z. Mathe, L. Buhler, M. Chilcott, P. Gervaz, J.-F. Egger and Ph. Morel, "Paraganglioma of the Ampulla of Vater: A Potentially Malignant Neoplasm,” Scandinavian Journal of Gastroenterology, Vol. 39, No. 3, 2004, pp. 291-295. doi:10.1080/00365520310007503

[14] T. Nagai, R. Torishima, H. Nakashima, J. Tanahashi, M. Iwata, H. Okawara, S. Yokoyama, R. Sato, K. Murakami and T. Fujioka, "Duodenal Gangliocytic Paraganglioma Treated with Endoscopic Hemostasis and Resection," Journal of Gastroenterology, Vol. 39, No. 3, 2004, pp. 277-283. doi:10.1007/s00535-003-1289-2

[15] Y. Okubo, T. Yokose, M. Tuchiya, A. Mitua, M. Wakayama, C. Hasegawa, D. Sasai, T. Nemoto and K. Shibuya, "Duodenal Gangliocytic Paraganglioma Showing Lymph Node Metastasis: A Rare Case Report," Diagnostic Pathology, Vol. 5, 2010, p. 27. doi:10.1186/1746-1596-5-27

[16] G. Schürmann, U. Raeth, B. Wiedenmann, H. Buhr and C. Herfarth, "Serum Chromogranin A in the Diagnosis and Follow-Up of Neuroendocrine Tumors of the Gastroenteropancreatic Tract," World Journal of Surgery, Vol. 16, No. 4, 1992, pp. 697-701. doi:10.1007/BF02067361 